\title{
Hand Held Farming
}

\author{
K.Jayanthi \\ Assistant Professor \\ Department of Electrical and \\ Electronics Engineering \\ Mepco Schlenk \\ Engineering College, \\ Sivakasi, India
}

\author{
M.Kirubasankar, \\ T.M.Arun Ramanath \\ Department of Electrical and \\ Electronics Engineering \\ Mepco Schlenk Engineering \\ College, \\ Sivakasi, India
}

\author{
C.Ruban Karthik, \\ .R.Sivasubramanian \\ Department of Electrical and \\ Electronics Engineering \\ Mepco Schlenk Engineering \\ College, Sivakasi \\ India
}

\begin{abstract}
The advancement of technology rises day by day ever since man learnt to create fire. Agriculture is being practiced by man over hundreds of centuries, the art of cultivation which is being told was on classical way as much as the development in technology towards science and engineering, uproots day by day in all the fields, A long time ago where a room sized machines with copper lines which are laid over hundreds of kilometers for communication, Now a simple hand sized device which we used to call as mobile has become more easy and comfy for the communication, Meanwhile the rising technologies like Nanotechnology, Intelligent Robotics, Three dimensional data storage, Power generation and transmission via smart grids, etc. have taken the ease of work and comfort ability to a great distant wherever and whatever the domain they were applied ,As the art of food production has also seen a lot of mile stones in recent decades. The application engineering Principles towards the statistical agriculture will promote the ease of farming. The process of cultivation takes a year long process right from the land setting (Ploughing) till the harvesting as of a general way and that involves frequent monitoring and protection issues of the field, as well as maintenance of farm with classical methods of crop growth using proper fertilizers, insecticides and pesticides accordingly, this process takes a lot of man-power using the classical methods as the Farm Laboring practice is out dated, the practice of farming on a large scale needs a major labor force and the method also is tedious. Here, In our protocol we establish a sequence of modules that assist the classical method of farming to a modernized way.
\end{abstract}

Key Words: Ploughing, Shredder, Arduino, sprayer, hover, GSM, Android etc.

\section{INTRODUCTION}

Hand Held Farming, where the complete monitoring and controlling process of the entire farm to a handed device the "Mobile", using this the irrigation of the land is completely taken by care by a mobile as any remote distance ( A person can monitor and control his irrigation system of his farm in Sivakasi, while travelling to Chennai) as the mobile communication takes place via satellites, Also for the security issues, we suggest a smart sensing fence, which mitigates trespassing and the animal intrusion, a frequent issue in hilly areas, this fence has modernized alarm system that prevents the entry and any level of trespassers. Moreover instead of using a heavy manned land roving vehicles like tractors ,a heavy and remote accesses land roving robot can be used for the purpose of Ploughing and Harvesting, which is economical, efficient and pollution free as of the use of vehicles, And ,Finally a Farm hover which is a low cost aviation robot used to spray pesticides, insecticides ,fertilizers to a land a very large
Scale just by controlling it by a remote, this can also be used for monitoring purpose, All these protocol are low cost and highly efficient in the control and monitoring of classical agricultural method at very affordable rates. This might take the agriculture to a great extent as the ease of work here is great than the classical way.

\section{GENERAL OVER VIEW}

This project comprises of Four Modules all together and interconnected

* Module -1 : Control and Monitoring of Farm field

- Manual Mode

- Automatic mode [5].

* Module -2: Smart Fencing System

* Module -3: Farm Hover ( Pesticide Spraying Robot)

* Module -4 : Land Rover ( Ploughing /Harvesting /Fertilizer Spraying Robot). 


\section{CONTROL AND MONITORING:- ( MANUAL MODE)}

The control and monitoring of the farm was completely taken down on to a mobile where the major components used are Microcontroller Board (Arduino),GSM Module, Pumps , Moisture sensors , Water level indicators and Rain Sensor.

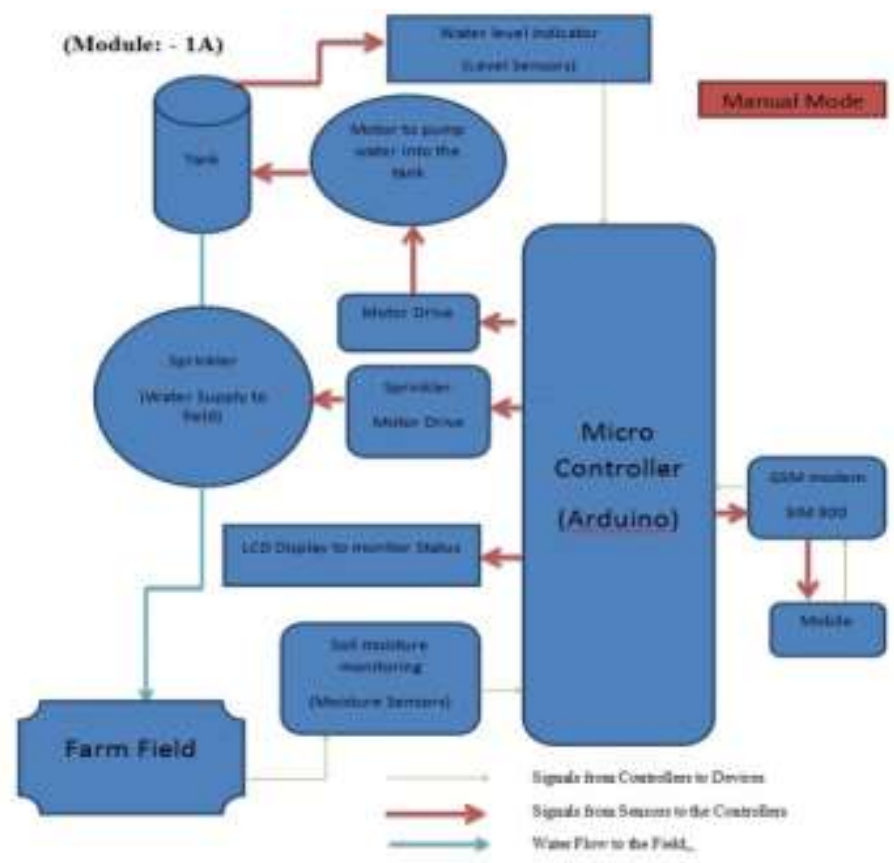

Fig 1.1 (Block diagram of operation)

Here the moisture sensors senses the soil moisture at all times as the farm field is to be wet at all times during early stages of farming, the sensor senses the signal and reports that to the controller, In case of any dry sensations the controllers provokes message to the users mobile via GSM module, User then decides whether to on the motor ( Sprinkler motor ) or not to, This sequence is given by input message to the controller to on the motor via the GSM module . [3]

Also the main water storage plant, which has a water level indicator, that senses the amount of water in the reserve and reports to the user on the account if the level is much low than the sensible level, here again a message is sent to the user's mobile about the indication and the user can retrieve i.e. on the motor by sending the authenticated signal to the controller via GSM, This module is extended with an ANDROID application for GSM based SMS communication.[6]

\section{CONTROL AND MONITORING:- ( AUTOMATIC MODE):}

Here the moisture sensors senses[6] the soil moisture at all times as the farm field is to be wet at all times during early stages of farming, the sensor senses the signal and reports that to the controller, In Case of any abnormalities on the sensing the sensors sends the signal to the controllers. The controller then sends the signal to the mobile about the condition and automatically turns on the Sprinkler motor to water the field, Also the main water storage plant, which has a water level indicator that senses the amount of water in the reserve and reports to the controller about the condition and the controllers report the user's mobile about the condition and also turns the motor to fill the reserve. [3]

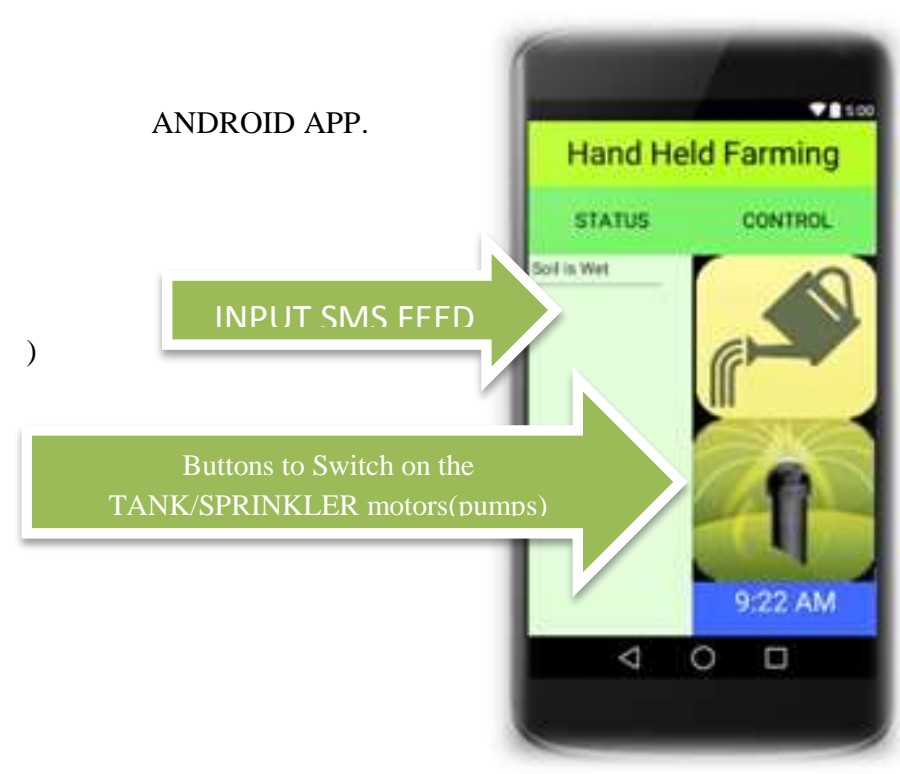

Fig1.2: (Layout of Android app to control/Monitor irrigation)

\section{SMART FENCING SYSTEM:}

Fencing System ensures the security of the farm with external intrusion from any trespasser and intruding animals (Dogs, Elephants etc.).The major components used in this module are Microcontroller (Arduino), GSM module, Proximity Sensors, Tactile Sensor, Range sensor, Alarm Trigger Systems, Smoke fire Trigger systems. As the proximity and Tactile sensors senses any object to its vicinity, as that's being reported to the controller and that in turn is informed to the user's mobile about the intrusion, and in case of a heavy sensing by the tactile sensors, the controllers triggers the alarm system and also ignites the smoke fire system, in case of any intrusion of animals . 
International Journal of Science and Engineering Applications

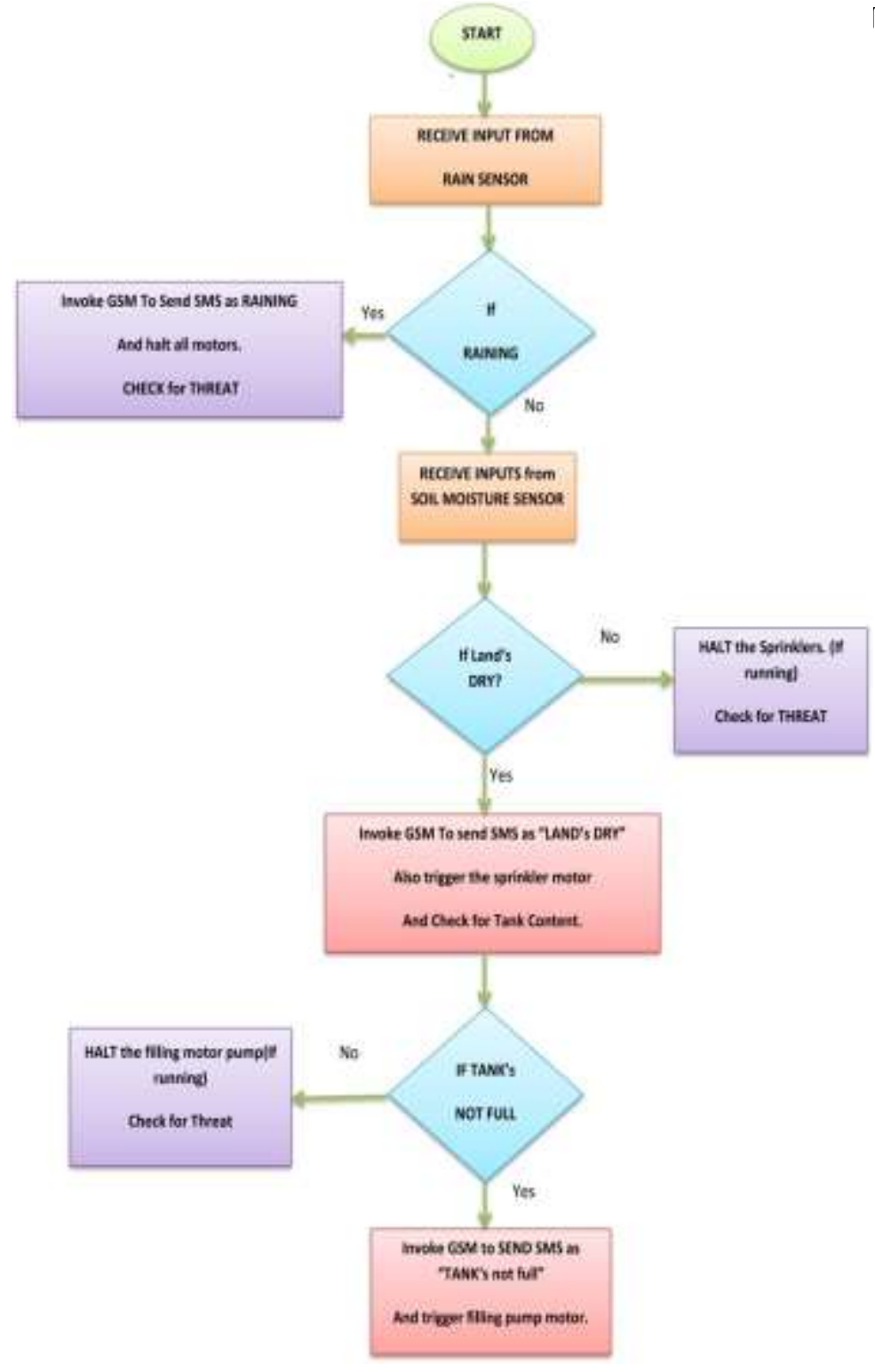

Fig 1.2a (Schematic Flow of Control of irrigation)

Also these Fences has a Range response system that reports the activity while using the module 3 \& 4 , Land rover and Farm hovers while working on the field their range and extent of the work is reported by these sensors ,they also sends feed back to the endpoints in the field.

\section{FARM HOVER}

As far as our smart hand held system of farming is concerned, this module FARM HOVER forms the integral part of our proposal. The following is the main constraint focused, PESTICIDE/FERTILIZER SPRAYING using farm hover via air. Our farm hover module takes the sole responsibility in establishing the above functions in a prejudiced manner. As far as our
-2319-7560 (Online)

project is concerned, this module comes with four main components as stated below: Microcontroller chip (ARDUINO UNO R2), Radio frequency RECEIVER and TRANSMITTER , Spray Canister, FARM HOVER with three Motion motors vertical axis motor, horiozontal axis motor, fly wheel motor and the spray pump.

In conjunction to the term micro controller, this component is mainly concerned with the entire controlling of the rover system. ARDUINO UNO R2 invoking ATMEGA 328P Chip is sole responsible for controlling the entire rover. UNO board is predominantly coded towards motion of the land rover. The receiving and transmitting circuits (RFID TRANSMITTER \& RECEIVER) are controlled in a programmed sequence so as to ensure efficient rover

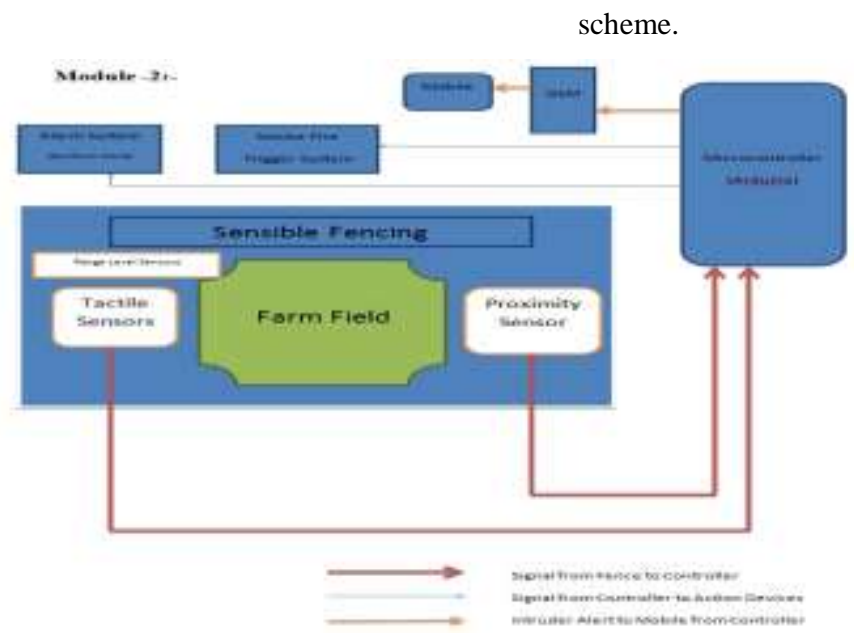

Fig: 1.3 (Block diagram of operation of smart fencing)

Being a transmitter, the RFID transmitter [4] transmits the inputted signals as Radio frequency waves with prescribed frequency range. And the receiver being in this frequency range receives the inputted signals from the transmitter through air as radio frequency, hence forming a wireless loop. This RFID component is highly ensured with transmitting the signal wirelessly.

\subsection{SPRAY CANNISTER:}

As the name implies, this component is highly responsible for spraying fertilisers from air. While the farm hover is in air under flying motion, this spray 
canister is excited to spray the fertilisers via air so as to ensure efficient spraying by cause of copter action.

\subsection{HOVER:}

This component is similar to a fly copter or some sort of air drone, controlled manually by remote action through distant ARDUINO encoded RFID transmitter. The construction is similar to a Mini copter

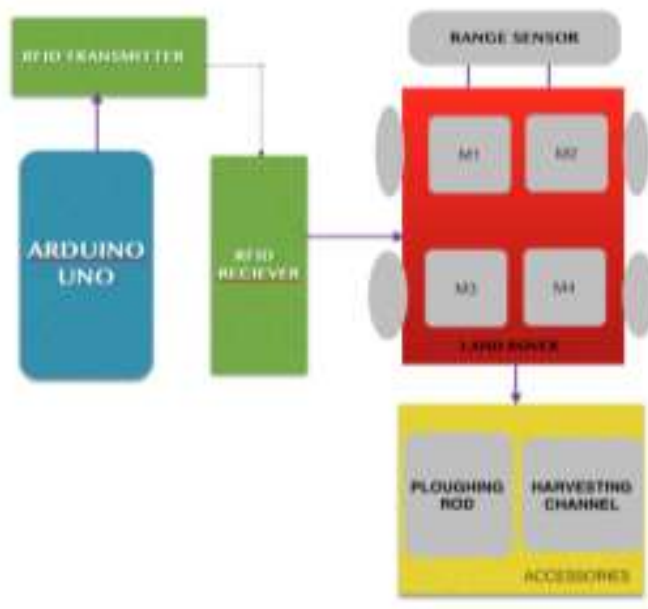

or an Air drone with fertiliser spray canister. The micro controller controls the flying motion of farm hover by controlling three motors as follows

Fig: 1.4: (Block diagram of LAND ROVER)

\subsection{VERTICAL AXIS MOTOR:}

Fig 1.5 (Typical Prototype of LAND ROVER)

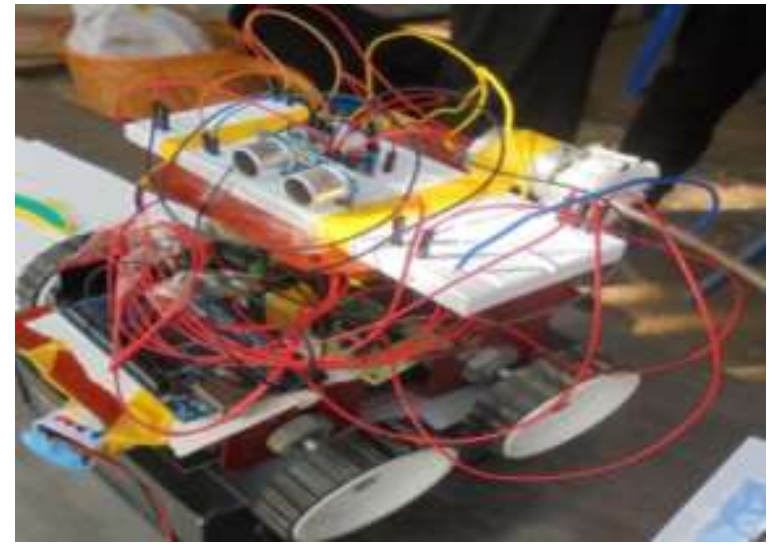

This motor ensures the upward movement of the farm hover. Whenever a copter or an air drone flies perpendicularly upwards, there is an up thrust motor
Under operation. It consists of a fly wheels which rotates in synchronism with the motor thus resulting in the generation of an upward thrust leading to the initial upward erection of the hover.

\subsection{HORIZONTAL AXIS MOTOR:}

This motor is responsible for the rotation or left-right motion of the hover and rotates in synchronism with the upthrust motor.

\subsection{FLYWHEEL MOTOR:}

This is the rear motor which helps in forward pushing of the motor which also rotates in synchronism with the other two motors.

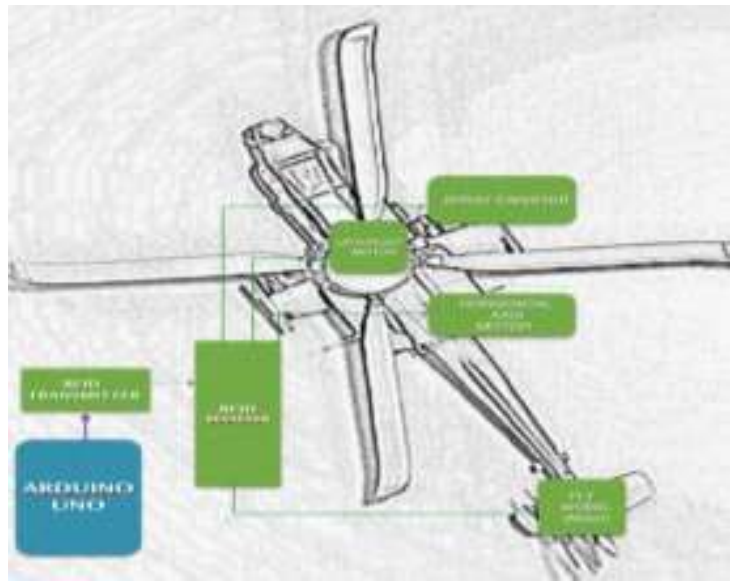

Fig: 1.5 (Block diagram of FARM HOVER)

\section{LAND ROVER}

Being a smart hand held system of farming, this module LAND ROVER forms the master piece of our proposal. The following are the two main constraints focuses, PLOUGHING the field so as to prepare it for farming, HARVESTING after farming, Our land rover module takes the sole responsibility in establishing the above two functions in a prejudiced manner. Also with an accessory like a linear pump attached to this UAV the fertilizer can easily be sprayed to the stems of very tough crops like corns etc. As far as our project is concerned, this module is made with four main components as stated below, Microcontroller chip (ARDUINO UNO R2), Radio frequency RECEIVER and TRANSMITTER, Ploughing/harvesting rover with RAMGE SENSOR, Accessories including, Harvesting channel, Ploughing rod and Spraying Pump. 
In conjunction to the term micro controller, this component is mainly concerned with the entire controlling of the rover system. ARDUINO UNO R2 invoking ATMEGA 328P Chip is sole responsible for controlling the entire rover. UNO board is predominantly coded towards motion of the land rover. The receiving and transmitting circuits (RFID TRANSMITTER \& RECEIVER) are controlled in a programmed sequence so as to ensure efficient rover scheme.

Being a transmitter, the RFID transmitter transmits the inputted signals as Radio frequency waves with prescribed frequency range. And the receiver being in this frequency range receives the inputted signals from the transmitter through air as radio frequency, hence forming a wireless loop. This RFID component is highly ensured with transmitting the signal wirelessly.

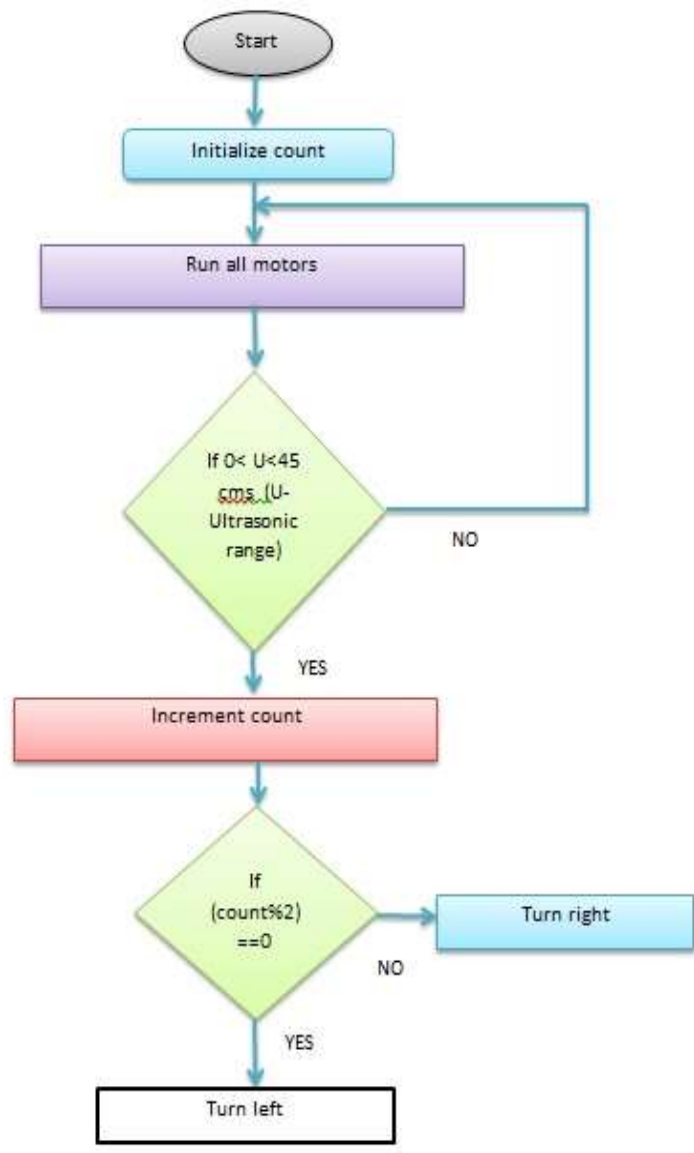

Fig: 1.5a (Schematic Flow for Automatic ploughing)

This component being the heart piece of the rover system comprises of four geared motors as mentioned in block diagram. The Motion of the gear motor is entirely controlled by the remote (either manually or automatically). Further efficient ploughing/harvesting is confined with the range sensor which senses the fencing boundary so as to prevent the rover from damage. The accessories include harvesting channel and ploughing rod which are rear end of the rover at a timely fashion whenever necessary.

\section{CONCLUSION:}

This idea suggests a best laid improvement of classical agricultural practice with modern technological advancements, As agriculture trends to be the low preferred occupation on this rising technological world ,the use of technology in this manner would definitely cause a strict rise in the GDP ( Gross Domestic Product ) of the agricultural field, moreover this practice helps to reduce the agricultural labor scarcity, thereby using some robotic based applications to do the basic agricultural amenities like ploughing, harvesting , spraying etc. The Control and monitoring of irrigation system is a best laid method for irrigating and monitoring the land this module can be used even (a person can monitor and control his irrigation system of his farm in Delhi, while travelling to Mumbai ) as the mobile communication takes place via satellites, Also for the security issues, we suggest a smart sensing fence, which mitigates trespassing and the animal intrusion, a frequent issue in hilly areas, this fence has modernized alarm system that prevents the entry and any level of trespassers. Moreover instead of using a heavy manned land roving vehicles like tractors , a heavy and remote accesses land roving robot can be used for the purpose of Ploughing and Harvesting, which is economical, efficient and pollution free as of the use of vehicles, And ,Finally a Farm hover which is a low cost aviation robot used to spray pesticides, insecticides, fertilizers to a land a very large scale just by controlling it by a remote, this can also be used for monitoring purpose, All these protocol are low cost and highly efficient in the control and monitoring of classical agricultural method at very affordable rates. This might take the agriculture to a great extent as the ease of work here is great than the classical way.

\section{REFERENCE:}

[1] Wireless Control of Irrigation System Operation From 3 Phase Induction Motor Fes By a Single Phase Supply Meenakshi Sundarajan n Engineering College : U.Deepa ,Meenakahsi- Department of Electrical and Electronics Engineering

[2] A Mobile Irrigation Lab for Water Conservation: Second Educational Development Field Data Danny H. Rogers, Gary Clark, Mahbub Alam, Robert Stratton, Steven Briggeman 
[3] Mobile Irrigation by Shobhan Kumar Asst.Prof, Dept of CSE shobhan.cs@ sahyadri.edu.in,

[4] GSM based Automated Irrigation Control using Raingun Irrigation System R.suresh, S.Gopinath, K.Govindaraju, T.Devika, N.SuthanthiraVanitha PG Student, Embedded System Technologies, Knowledge Institute of Technology, Salem, India, Assistant professors, Department of Electrical \& Electronics Engineering, Knowledge Institute of Technology Salem India4 Professor \& Head, Department of Electrical \& Electronics Engineering, Knowledge Institute of Technology, Salem, India

[5] Extremely Secured Remote accessed Irrigation system a Mini Project report by M.Kiruba Sankar, R.Sivasubramanian, C.RubanKarthik guided by Mrs.MeenaLakshmi Assistant Professor Mepco Schlenk Engineering College.www.scribd.com.

[6] Control of Irrigation Automatically By Using Wireless Sensor Network Rashid Hussain, JL Sahgal, Anshulgangwar, Md.Riyaj

[7] Wireless Watering New irrigation technologies from ARS can help conserve a vital resource, Wireless Watering on Agricultural Research/July 2007.

[8] GSM Based Irrigation Control and Monitoring System by GODFREY A. MILLS Computer Engineering Department, University of Ghana, 